\title{
Evaluating the impact of declining tsetse fly (Glossina pallidipes) habitat in the Zambezi Valley of Zimbabwe
}

Farai Matawa ${ }^{\mathrm{a}, \mathrm{b}}$ *, Amon Murwira ${ }^{\mathrm{a}}$, Peter M. Atkinson ${ }^{\mathrm{c}, \mathrm{d}, \mathrm{e}, \mathrm{f}}$

${ }^{a}$ Department of Geography and Environmental Science, University of Zimbabwe, P.O. Box MP167, Mount Pleasant, Harare, Zimbabwe

b Geo-information and Remote Sensing Institute, Scientific and Industrial Research and Development, 1574 Alpes Road, Hatcliffe, Harare, Zimbabwe

${ }^{c}$ Faculty of Science and Technology, Lancaster University, Lancaster, LA1 4YR, UK

${ }^{\mathrm{d}}$ School of Built and Natural Environment, Queen's University Belfast, Belfast, BT7 1NN Northern Ireland, UK

${ }^{\mathrm{e}}$ Geography and Environmental Science, University of Southampton, Highfield, Southampton, SO17 1BJ, UK

${ }^{\mathrm{f}}$ Institute of Geographic Science and Natural Resources Research, Chinese Academy of Sciences, 11A Datun Road, Beijing 100101, China 


\begin{abstract}
Tsetse flies transmit trypanosomes that cause Human African Trypanosomiasis (HAT) in humans and African Animal Trypanosomiasis (AAT) in animals. Understanding historical trends in the spatial distribution of tsetse fly habitat is necessary for planning vector control measures. The objectives of this study were (i) to test for evidence of any trends in suitable tsetse fly habitat and (ii) to test whether there is an association between trypanosomiasis detected from livestock sampled in dip tanks and local tsetse habitat in the project area. Results indicate a significant decreasing trend in the amount of suitable habitat. There is no significant correlation between trypanosomiasis prevalence rates in cattle and distance from patches of suitable tsetse habitat. The observed low trypanosomiasis prevalence and the lack of dependence on suitable tsetse fly habitat can be explained by the observed decreases in suitable tsetse habitat, which themselves are due to expansion of settlement and agriculture in North Western Zimbabwe.
\end{abstract}

\title{
Introduction
}

The tsetse fly (Glossina spp.) transmits trypanosomes that are responsible for causing Human African Trypanosomiasis (HAT) in humans and African Animal Trypanosomiasis (AAT) in animals (Holmes, 2013; Matawa et al., 2016, 2013). AAT results in increased rural poverty due to loss of livestock that represents a major source of livelihood (Holmes, 2013; Matawa et al., 2016). In this regard, well planned vector control and eradication measures can improve rural livelihoods through improved livestock production. Characterising temporal trends in the spatial distribution of tsetse flies may, thus, be used to guide the planning of future vector control and eradication measures (Dicko et al., 2014; Matawa et al., 2016, 2013).

Studies have shown that a reduction in the area of woody vegetation cover often results in reduction in the amount of suitable tsetse habitat in tsetse infested areas. This is due to the fact that loss of woody vegetation cover results in loss of both shade for tsetse flies and wildlife that provide the requisite blood meals for the tsetse flies (Kitron et al., 1996; Matawa et al., 2016; Munang'andu et al., 2012; Scoones et al., 2017). Therefore, this study evaluates likely 
trends and changes in suitable tsetse fly habitat in the light of environmental changes in the Zambezi valley of Zimbabwe.

Evaluating trends in time-series of spatial data on tsetse habitat in relation to the expansion of settlement and agriculture in the Zambezi valley is needed to explain trypanosomiasis prevalence in livestock in the light of human encroachment into wild-lands and plan tsetse control. According to Van Den Bossche et al. (2010) human encroachment can create settings in which tsetse have "disappeared" because of habitat loss, but where livestock may be bitten by tsetse flies at the edge of tsetse-infested protected wildlife zones, or settings in which the density of game animals is low and livestock constitute the main source of food for tsetse flies. Once livestock are the main source of blood meals for tsetse flies the transmission cycle changes from a sylvatic cycle to a domestic cycle (Van Den Bossche et al., 2010). Studies on ecosystem change and its effect on tsetse habitat dynamics have remained limited (Matawa et al., 2016). In addition, few studies have investigated the impact of demography, land tenure, agriculture and livestock-production systems and habitat fragmentation on tsetse epidemiology with detailed scientific evidence (Van Den Bossche et al., 2010). Although an understanding of the temporal trends in suitable tsetse habitat is critical in characterising the dynamics of Trypanosomiasis transmission, studies on evaluating such trends in change in suitable tsetse habitat and their impact on tsetse transmission cycles have remained limited (Van Den Bossche et al., 2010). This study evaluates the trends and changes in suitable tsetse habitat and explains the pattern of trypanosome prevalence in livestock (cattle and goats) samples in the Hurungwe area of Zimbabwe in the context of these changes in tsetse fly habitat.

Methods of evaluating trends in spatial time-series data include simple regression (Matawa et al., 2016), calculating percentage change (Diniz-Filho et al., 2010, 2009; Lawler et al., 2006; Pearson, 2006; Thuiller, 2004; Yates et al., 2010) and use of time-series analysis techniques such as the Mann-Kendall test (Best and Gipps, 1974; Hamed, 2008; Kendall, 1976; Kisi and Ay, 2014), Sen's slope (Kisi and Ay, 2014), and the Cox-Stuart test (Rutkowska, 2015). This study uses the Mann-Kendall test because it can account for serial correlation in the data (Holsten et al., 2009), can manage non-normality, and has a high asymptotic efficiency (Yan et al., 2013). The Cox-Stuart test is slightly weaker in comparison to the Mann-Kendall test (Rutkowska, 2015). Where earlier studies have analysed spatial-temporal trends in species habitat, the studies have focused more on calculating percentage gains or losses in the future, usually based on climate change forecasts (e.g., Diniz-Filho et al., 2010, 2009; Lawler et al., 2006; Pearson et al., 2006; Thuiller, 2004; and Yates et al., 2010). In contrast, this research 
evaluates temporal trends in suitable tsetse habitat in relation to changes in woody vegetation cover as measured by remotely sensed NDVI based on results from an ensemble model using the Mann-Kendall test for trends.

In this study, we test for any significant trend in suitable habitat based on an ensemble model derived from elevation and topographic position index (TPI) (temporary stable variables) and vegetation cover (dynamic variable) (Matawa et al., 2016). In addition, we test whether there is significant relationship between trypanosomiasis prevalence rates and distance from patches of suitable tsetse fly habitat. A statistically significant negative correlation between trypanosomiasis prevalence rates and Euclidean distance from patches of suitable tsetse fly habitat could indicate that high trypanosomiasis prevalence rates are clustered around patches of suitable tsetse fly habitat. This study is premised on the assertion that changes in vegetation cover over time due to expansion of settlement and agriculture may result in changes in suitable habitat for tsetse flies. Considering that elevation and TPI are largely temporally stable, the change in suitable tsetse habitat can be explained by a change in vegetation cover as NDVI is generally temporally dynamic (Matawa et al., 2016).

\section{Materials and Methods}

\section{Study area}

The study area is located in North Eastern Zimbabwe (Figure 1). The area straddles protected areas (including safari areas) and settled areas comprising large and small scale farming as well as the communal lands of the Zambezi Valley (Matawa et al., 2016). Communal lands are areas characterised by community land ownership (Sibanda and Murwira, 2012). The area is characterised by low and variable annual rainfall averaging between 450 and $650 \mathrm{~mm}$ per year and a mean annual temperature of $25^{\circ} \mathrm{C}$ (Matawa et al., 2016). It has two clearly defined seasons: a wet season from December to March and a long dry season from April to November (Baudron et al., 2010; Matawa et al., 2016). The climatic conditions, thus, make the study area suitable as habitat for tsetse (Matawa et al., 2016). The natural vegetation is mainly deciduous dry savannah (Baudron et al., 2010; Matawa et al., 2016; Sibanda and Murwira, 2012). The elevation ranges from $340 \mathrm{~m}$ to $1400 \mathrm{~m}$ (Matawa et al., 2016). According to Pender et al. (1997), 1997, areas below $1100 \mathrm{~m}$ are climatically suitable for tsetse. 


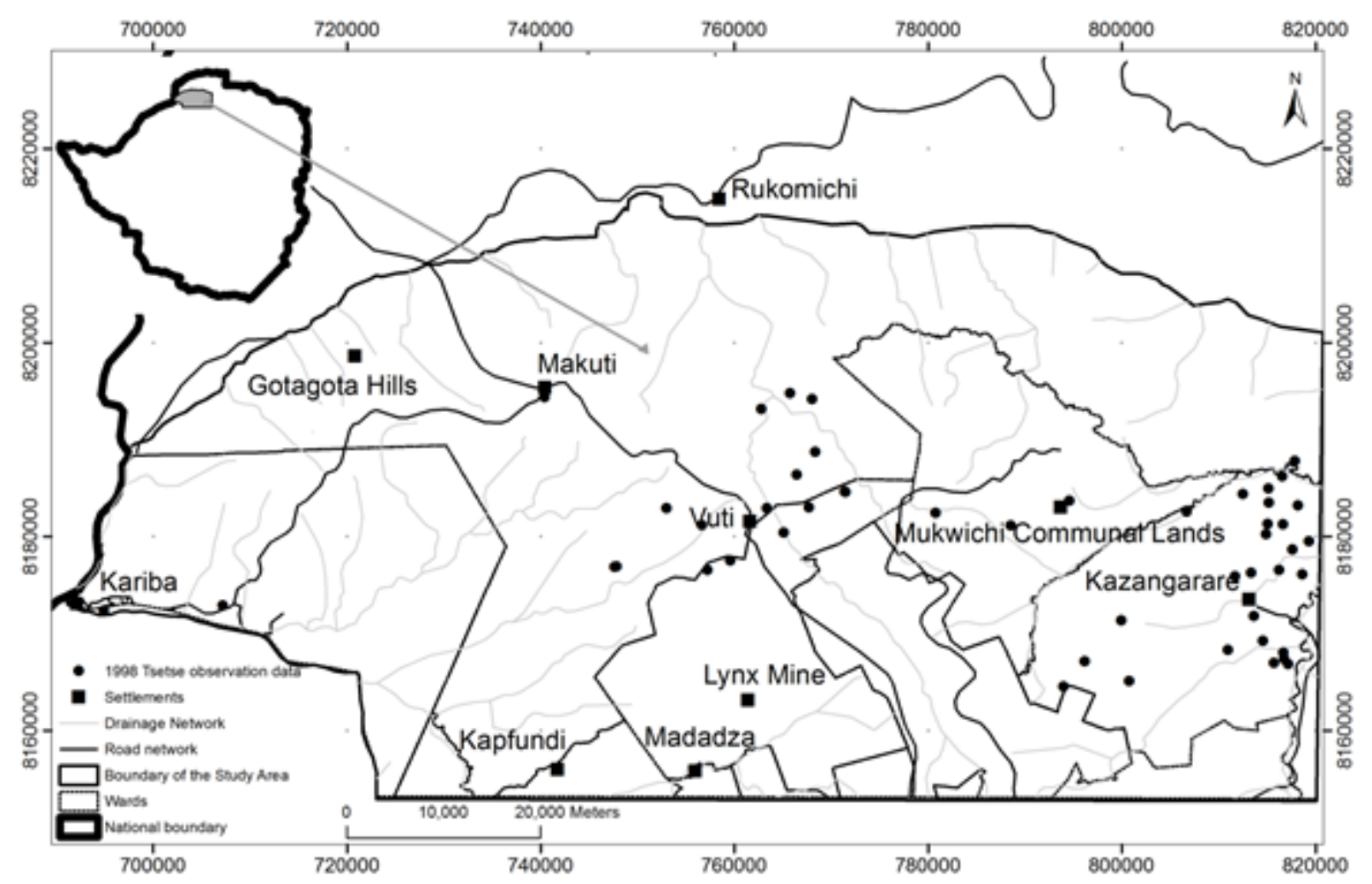

Figure 1: Location of the study area in Zimbabwe (Matawa et al., 2016).

\section{Species occurrence data}

Data on tsetse occurrence were extracted from tsetse fly trap records for the period 1994 to 2012. We used the 1998 dataset for training the model because it had a wider geographic spread and a larger number of presence records (50) (Figure 1) (Matawa et al., 2016). The tsetse fly trap records were collected by the Zimbabwe Department of Veterinary Services and Livestock Production, Tsetse Control Division in Harare (Figure 1) (Matawa et al., 2016). Historical data on trap locations in Zimbabwe were read and marked with pencil on 1:250,000 topographic map sheets. There is room for locational error in the data. In addition, tsetse flies were trapped using odour baited targets often placed in open areas. Therefore, there exists the possibility of trapping tsetse outside of their normal habitat.

\section{Environmental variables}

We downloaded cloud-free (less than $10 \%$ cloud) Landsat Thematic Mapper (TM), Enhanced Thematic Mapper (ETM) with a spatial resolution of $30 \mathrm{~m}$ and Operational land Imager (OLI) satellite sensor data made available at the USGS EROS Data Centre (http://lpdaac.usgs.gov/) to estimate vegetation greenness (Matawa et al., 2016). Satellite sensor data were collected for the period April to early-July (day 110 to day 199) (Matawa et al., 2016) for the years 1986, 
1991, 1993, 1998, 2002, 2007, 2008 and 2014. We focused on the period from end-April to early-July (post-harvest period) as all trees in the study area are still in full leaf then, while grass and crops are in the senesce stages thereby making it easier to isolate and explain the impact of land use/land cover changes (Matawa et al., 2016; Sibanda and Murwira, 2012). Vegetation greenness was estimated using the Normalised Difference Vegetation Index (NDVI). We calculated average NDVI based on available Landsat TM, ETM and OLI imagery between day 110 and day 199 of each year. We selected years with at least two or more images for the analysis (Matawa et al., 2016).

\section{Ensemble Modelling}

We used the Biomod 2 toolkit in the R statistics software to develop a stable and temporary dynamic tsetse distribution model using the ensemble mean and the ensemble weighted mean modelling techniques. Our initial model (Matawa et al., 2016) was developed based on the Maximum Entropy technique (Phillips et al., 2006). We used remotely sensed NDVI, an indicator of vegetation productivity and greenness as a temporally dynamic variable, and topographic variables (i.e., altitude and TPI) as temporally stable variables (Matawa et al., 2016). The model was developed based on tsetse observation data and NDVI of 1998. The model was applied to years with available NDVI data (i.e. 1986, 1991, 1993, 2002, 2007, 2008 (Matawa et al., 2016) and 2014). Our ensemble model considered all models which produced an AUC greater than 0.7 and had a consistent relationship between AUC and TSS based on five runs. We selected a model with at least four data points falling in the bounding box in the top right corner of the scatterplot (Figure 2). In this case we selected the Maximum Entropy (Maxent), Generalized Linear Model (GLM), Random Forest (RF) and Flexible Discriminant Analysis (FDA) models to build the ensemble. The models were combined based on the following weights: Maxent (0.255), GLM (0.255), FDA (0.237) and RF (0.252). 


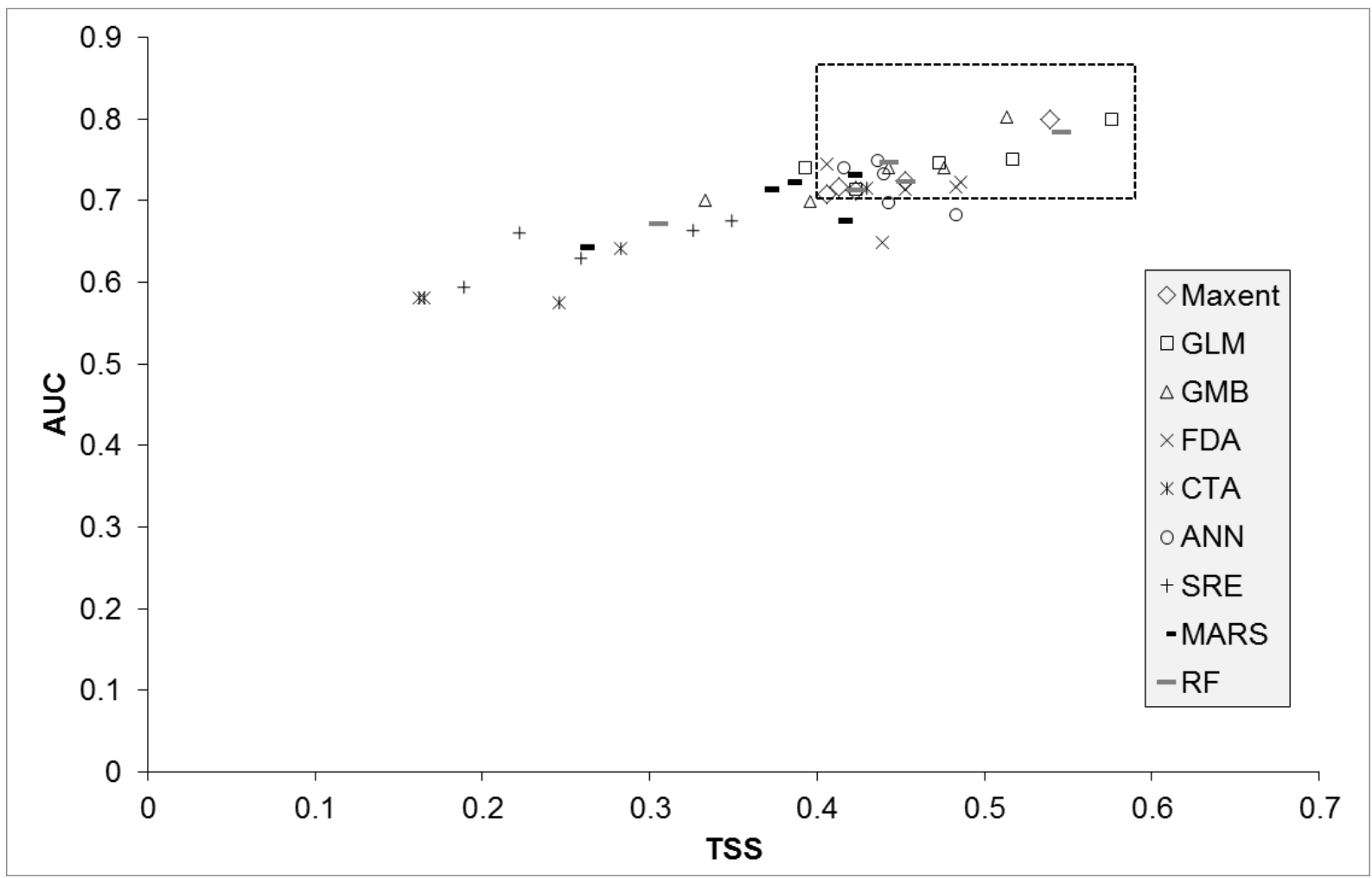

Figure 2: Relationship between AUC and TSS used to select models for inclusion into the Ensemble model.

\section{Evaluating model accuracy}

We evaluated model performance based on the AUC (Peterson et al., 2007; Phillips and Dudik, 2008) and TSS (Allouche et al., 2006), using independent test datasets to validate the models (30\% of the species observation data (Matawa et al., 2016, 2012). AUC can be classified as insufficient (0.50-0.60); poor (0.60-0.70); average (0.70-0.80); good (0.80-0.90) and excellent (0.90-1) (Parolo et al., 2008). TSS ranges from -1 to +1 , where +1 indicates perfect agreement and values of zero or less indicate a performance no better than random (Allouche et al., 2006).

Table 1 shows that the Ensemble models have an AUC value greater than 0.9 and a TSS value greater than 0.7. The model performed better than random suggesting our model based on elevation, TPI and NDVI can be used to model and track changes in the spatial temporal distribution of suitable tsetse habitat.

Table 1: Testing the accuracy of different models

\begin{tabular}{ll|l}
\hline Algorithm/ Ensemble weighted mean & Ensemble mean \\
Statistic & & \\
\hline
\end{tabular}




\begin{tabular}{llll|lll}
\hline & Statistic & Sensitivity & Specificity & Statistic & Sensitivity & Specificity \\
\hline TSS & 0.771 & 78.571 & 98.3 & 0.771 & 78.571 & 98.5 \\
& & & & & & \\
\hline AUC & 0.936 & 78.571 & 98.6 & 0.936 & 78.571 & 98.6 \\
& & & & & & \\
\hline
\end{tabular}

\section{Evaluation of spatial-temporal trends in suitable habitat between 1986}

\section{and 2014}

To test whether there was a significant monotonic downward trend in tsetse habitat suitability between 1986 and 2014, we applied the Mann-Kendall trend test in R to the time-series data. Prior to calculating the Mann-Kendall statistic, we tested whether the suitable habitat values were serially correlated (autocorrelation and partial autocorrelation). We wanted to determine whether or not to use the Mann-Kendall test with block bootstrapping to account for serial correlation present in the modelled suitable habitat time-series data. In this research, we applied the Mann-Kendall test without block bootstrapping as our data did not show significant autocorrelation and partial autocorrelation. The assumption is that if most of the vertical spikes produced are within the lower and upper limit there is no autocorrelation and partial autocorrelation and, therefore, there may be no need to correct for autocorrelation.

The Mann-Kendall test is formulated as follows:

$$
T=\frac{S}{D}
$$

Where $S=\sum_{i<j}(\operatorname{sign}(x[j]-x[i]) * \operatorname{sign}(y[j]-y[i]))$

$$
D=n(n-1) / 2
$$

$S$ is the score and $D$ is the denominator (the maximum possible value of $S$ ) (Best and Gipps, 1974; Kendall, 1976). The Mann-Kendall test is ideal for trend analysis because it is robust to departures from normality (Blain, 2013) and is less sensitive to outliers (Hamed, 2008).

\section{Analysing the pattern of trypanosomiasis prevalence in the study area}

Livestock blood samples were taken at each dip tank in the project area to screen for trypanosomiasis parasites. We calculated the prevalence rate as the number of infected livestock divided by the total number of livestock screened at each dip tank. Goat and cattle 
prevalence was analysed separately. We also overlayed the suitable habitat with locations of dip tanks to establish their spatial relationship. In addition, we calculated the Euclidean distance from each patch of suitable tsetse fly habitat and related it to the location of trypanosomiasis infections in cattle. Firstly, we tested whether the trypanosomiasis prevalence and distance from patches of suitable habitat data were normally distributed and found the distance data to be non-normally distributed. We then used Spearman's rank correlation coefficient to test the relationship between the two variables. This was done to establish whether or not there is a dependence between the spatial pattern of trypanosomiasis and suitable tsetse fly habitat in the Mukwichi communal lands.

\section{Results}

\section{Trends in suitable habitat between 1986 and 2014}

We observed that the amount of suitable G. pallidipes habitat decreased between 1986 and 2014, associated with a decrease in woody vegetation cover (Figure 3). The results (Table 2) illustrate a significant decrease in the amount of suitable habitat as shown by negative Tau values based on the results of the ensemble mean and ensemble weighted mean (Tau $=-0.571$, $p=0.048)$.

We observed that although there is slight increase in the amount of suitable tsetse habitat between 2007 and 2014 based on the results of the ensemble mean and ensemble weighted mean model (Figure 3), the positive trend is not statistically significant (Tau $=0.333 ; p=$ 0.602). Instead, we observed that there was a decrease in the amount of suitable habitat in the Mukwichi communal area between 1986 and 2014. 


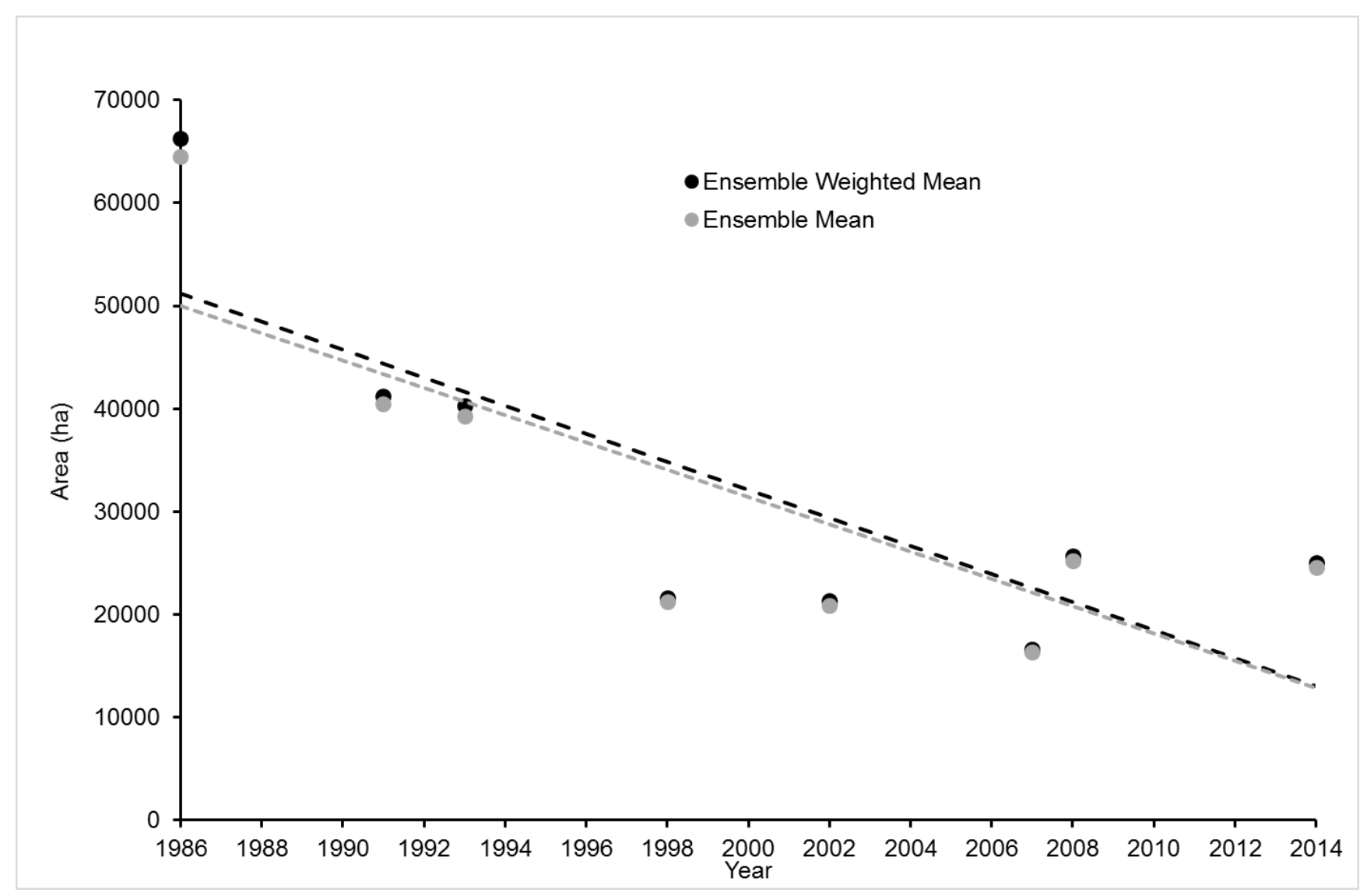

Figure 3: Change in suitable habitat ( $y$-axis) through time ( $x$-axis) for $G$. pallidipes based on the results of the ensemble modelling technique.

Table 2: The estimated trend in suitable tsetse habitat over time based on the Mann-Kendall Test.
Model/ value
tau
$P$-value

\begin{tabular}{lll}
\hline Ensemble Mean & -0.571 & $0.048^{*}$ \\
\hline Ensemble Weighted mean & -0.571 & $0.048^{*}$
\end{tabular}

* statistically significant monotonic downward trend between 1986 and 2014

The agreement between the Ensemble Mean and Ensemble Weighted Mean models ranges between $99.71 \%$ and $99.95 \%$ (Table 3), thereby, showing that they were modelling almost similar pixels as suitable G. pallidipes habitat.

Table 3: Agreement between the ensemble mean and ensemble weighted mean models (\%).

\begin{tabular}{|c|c|c|c|c|c|c|c|c|c|}
\hline Model & \multicolumn{9}{|c|}{ Ensemble weighted mean } \\
\hline 氠 & Year & 1986 & 1991 & 1993 & 1998 & 2002 & 2007 & 2008 & 2014 \\
\hline
\end{tabular}




\begin{tabular}{|c|c|c|c|c|c|c|c|c|}
\hline 1986 & 99.71 & & & & & & & \\
\hline 1991 & & 99.87 & & & & & & \\
\hline 1993 & & & 99.84 & & & & & \\
\hline 1998 & & & & 99.94 & & & & \\
\hline 2002 & & & & & 99.93 & & & \\
\hline 2007 & & & & & & 99.95 & & \\
\hline 2008 & & & & & & & 99.92 & \\
\hline 2014 & & & & & & & & 99.92 \\
\hline
\end{tabular}

\section{Pattern of trypanosomiasis prevalence in the study area}

Positive trypanosomiasis infections were detected from cattle and goat blood samples collected at dip tanks namely Chingwena (10\% of cattle were infected with Trypanosomiasis brucei ( $n$ =40)), Chitindiva (7.5\% of cattle were infected $(n=40$, evidence of $T$. brucei and Trypanosomiasis vivax)), Mafuwa (13\% of cattle $(n=23)$ and one goat was identified with a dual infection), Mayamba (1.25\% of cattle $(n=80)$ were infected with $T$. vivax) and Village 22 (5\% of cattle ( $n=40$, evidence of T. brucei and T. vivax) in 2014 (Figure 4 and Figure 5). Our results show that Mafuwa, with the highest prevalence rate (13\%), falls within a patch of suitable tsetse fly habitat. This indicates that tsetse flies and the trypanosomiasis they transmit still persist in the communal areas south of the game fence although the prevalence rates are low. There is no statistically significant negative correlation between trypanosomiasis prevalence rates in cattle and Euclidean distance from patches of suitable tsetse fly habitat $(r=$ $-0.309, p=0.304)$. 


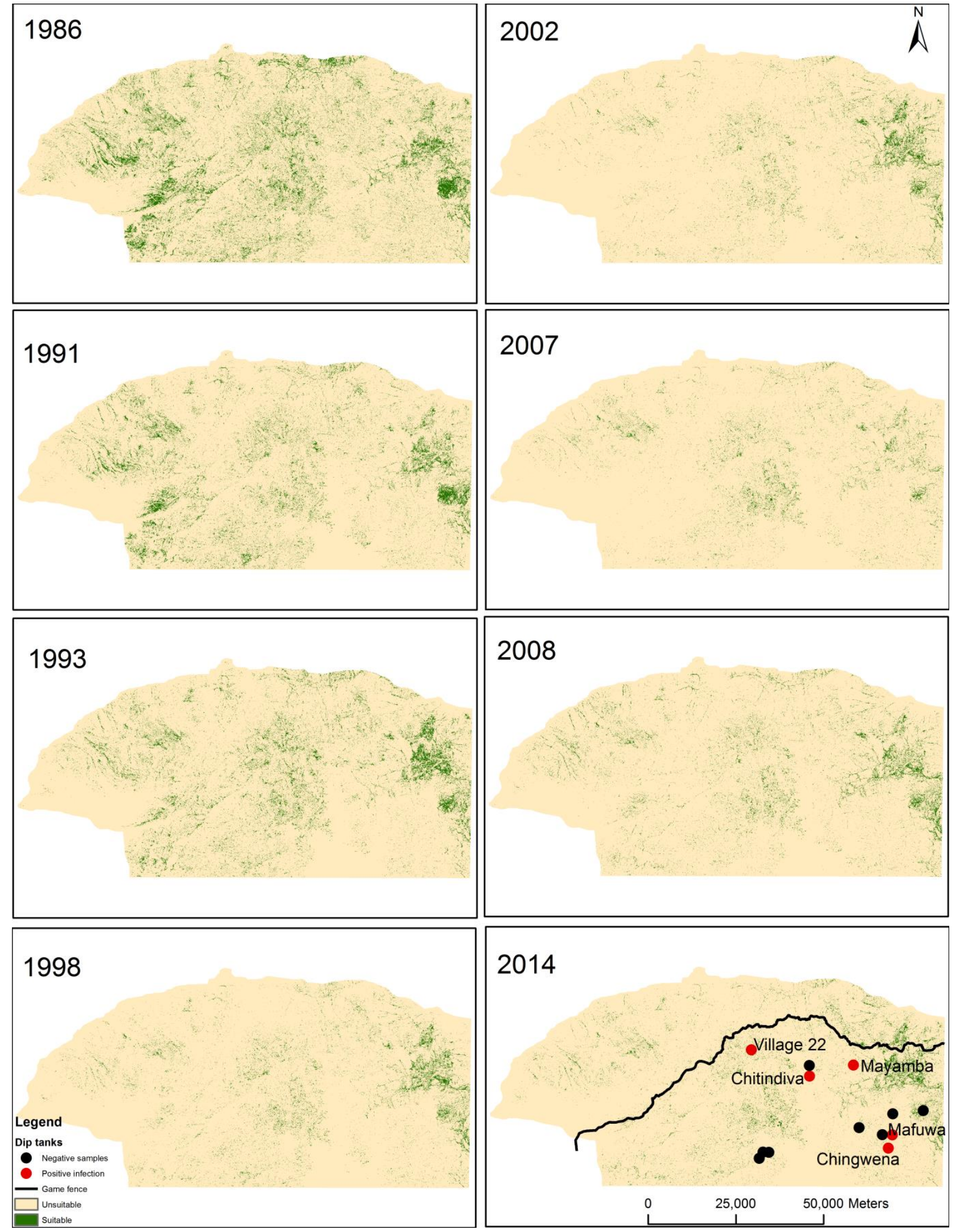

Figure 4: Time-series variation in the amount of suitable tsetse habitat based on the ensemble weighted mean algorithm between 1986 and 2014. 


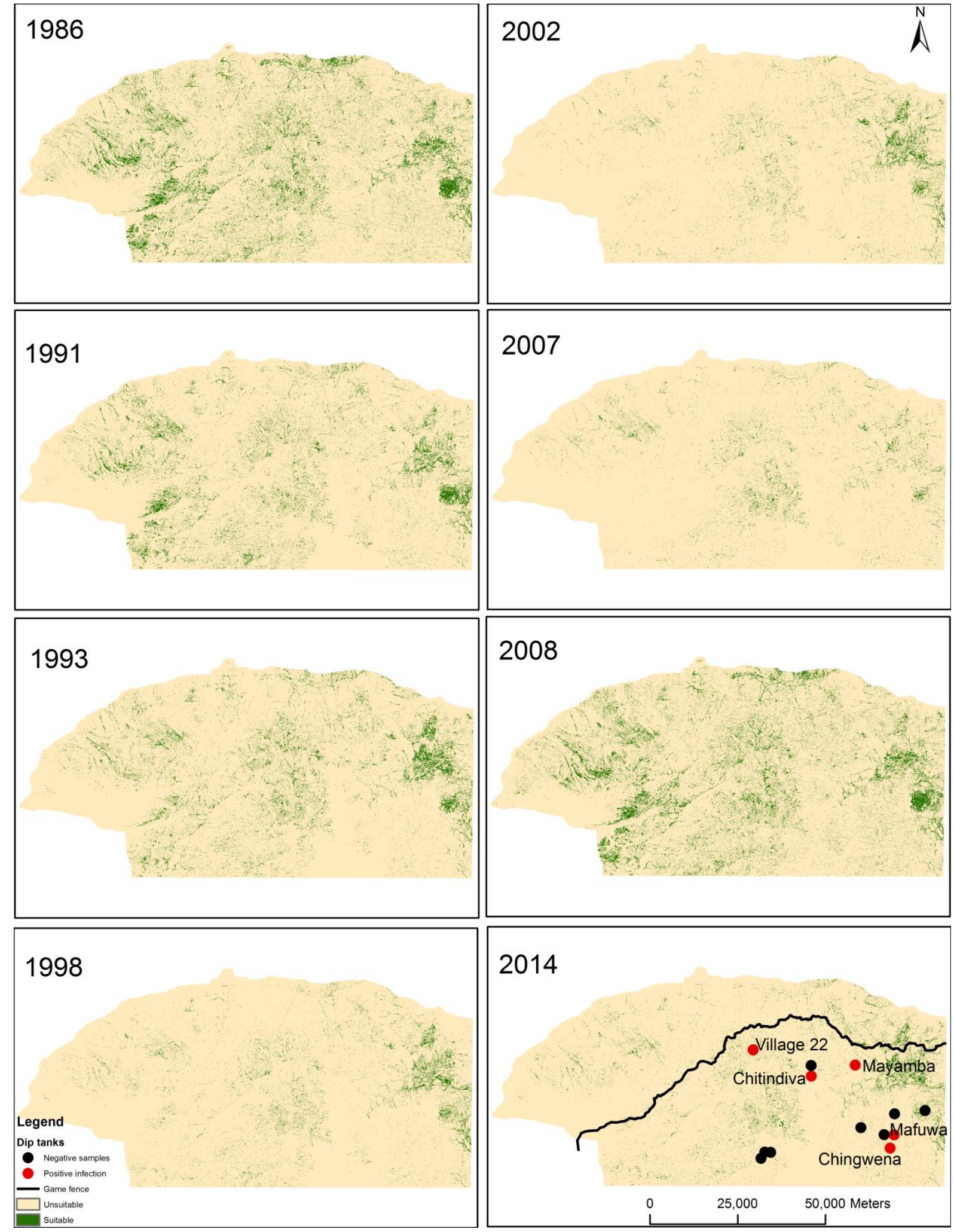

Figure 5: Time-series variation in the amount of suitable tsetse habitat based on the ensemble mean algorithm between 1986 and 2014. 


\section{Discussion}

Results in this study indicate a general and statistically significant decreasing trend in suitable G. pallidipes habitat in the Zambezi valley between 1986 and 2014. The decreasing trend in suitable habitat is consistent with the hypothesis that there was a decrease in suitable tsetse habitat between 1986 and 2014. The results are consistent with the findings of Matawa et al., (2016) and Scoones et al., 2017 that there was a decrease in suitable tsetse habitat associated with a decrease in vegetation cover due to the expansion of settlement and agriculture. Thus, we confirm that loss of woody vegetation cover due to expansion of settlement and agriculture across time has resulted in a significant decrease in the amount of suitable tsetse fly habitat.

The decrease in suitable tsetse habitat due to expansion of agriculture explains the low trypanosomiasis prevalence in livestock in the Mukwichi communal lands. Most of the dip tanks did not record positive trypanosomiasis infections in livestock with the highest prevalence rate being 13\% (Mafuwa, $n=23$ ). AAT was evident in cattle blood samples and one goat blood sample taken from dip tanks in the Muckwichi communal lands. Our results indicate that there is no statistically significant relationship between trypanosomiasis prevalence rates in cattle and distance from patches of suitable tsetse fly habitat. Thus, our results indicate that high trypanosomiasis prevalence rates are not clustered around patches of suitable tsetse fly habitat. The pattern of distribution in relation to patches of suitable tsetse fly habitat suggest 'incidental' or 'by chance infection' associated with situations where livestock are bitten by tsetse flies when they come into contact with tsetse flies in suitable habitats such as tsetse-infested protected wildlife zones as suggested by Van Den Bossche et al. (2010). Most sites are within the $250 \mathrm{~m}$ distance from suitable patches of tsetse habitat which can be covered by a tsetse fly in a single day (DeVisser et al., 2010). Thus, the infections and prevalence rates can be explained by livestock and tsetse fly movement across patches of suitable habitat.

Our study differs from earlier studies in that we used ensemble models built based on objective criteria, to track changes in suitable tsetse habitat over a long period (i.e. 28 years from 1986 to 2014; with eight individual years spanning the 28-year period), making the results reliable. In addition, we used remotely sensed $30 \mathrm{~m}$ resolution NDVI as the dynamic variable to track and evaluate the trend in suitable tsetse habitat between 1986 and 2014 using ensemble models. We used NDVI as the only dynamic variable in the model to isolate and explain the role of vegetation cover in shaping the distribution of tsetse habitat (Matawa et al., 2016). 
The results can inform tsetse control planning initiatives where we recommend that tsetse fly control initiatives be more target, for example, targeting a buffer zone along the game fence and in patches of modelled suitable tsetse habitat in the communal areas to reduce the chance of trypanosomiasis infection in livestock. Monitoring traps can be placed in patches of suitable habitat to so that reinvasion can be detected at an earlier stage.

Our results allow a better understanding of the relationship between tsetse presence and decrease in woody vegetation cover measured using remotely sensing and the impact of the decrease in woody vegetation cover on trypanosomiasis prevalence. In addition, the results are reliable such that they can inform targeted vector control as evidenced by the consistence of the results thereby optimizing vector control.

\section{References}

Allouche, O., Tsoar, A., Kadmon, R., 2006. Assessing the accuracy of species distribution models: prevalence, kappa and the true skill statistic (TSS). J. Appl. Ecol. 43, 1223-1232. doi:10.1111/j.1365-2664.2006.01214.x

Ashraf, U., Peterson, A.T., Chaudhry, M.N., Ashraf, I., Saqib, Z., Ahmad, S.R., Ali, H., 2017. Ecological niche model comparison under different climate scenarios: A case study of Olea spp. in Asia. Ecosphere 8. doi:10.1002/ecs2.1825

Baudron, F., Corbeels, M., Andersson, J.A., Giller, K.E., Sibanda, M., 2010. Delineating the drivers of waning wildlife habitat: The predominance of cotton farming on the fringe of protected areas in the Mid Zambezi valley, Zimbabwe. Biol. Conserv. 144, 1481-1493.

Best, D.J., Gipps, P.G., 1974. Algorithm AS 71: The Upper Tail Probabilities of Kendall's Tau. Appl. Stat. 23, 98-100.

Blain, G.C., 2013. The Mann-Kendall test: the need to consider the interaction between serial correlation and trend. Acta Sci. 35, 393-402. doi:10.4025/actasciagron.v35i4.16006

DeVisser, M.H., Messina, J.P., Moore, N.J., Lusch, D.P., Maitima, J., 2010. A dynamic species distribution model of Glossina subgenus Morsitans: The identification of tsetse reservoirs and refugia. Ecosphere 1, 1-21. doi:10.1890/ES10-00006.1

Dicko, A.H., Lancelot, R., Seck, M.T., Guerrini, L., Sall, B., Lo, M., Vreysen, M.J.B., 2014. Using species distribution models to optimize vector control in the framework of the tsetse 
eradication campaign in Senegal. Proccedings Natl. Acad. Sci. 111, 10149-10154. doi:10.1073/pnas.1407773111

Diniz-Filho, J.A.F., Bini, L.M., Rangel, T.F., Loyola, R.D., Hof, C., Nogues-Bravo, D., Araújo, M.B., 2009. Partitioning and mapping uncertainties in ensembles of forecasts of species turnover under climate change. Ecography (Cop.). 32, 897-906. doi:10.1111/j.16000587.2009.06196.x

Diniz-Filho, J.A.F., Ferro, V.G., Santos, T., Nabout, J.C., Dobrovolski, R., De Marco, P.J., 2010. The three phases of the ensemble forecasting of niche models: geographic range and shifts in climatically suitable areas of Utetheisa ornatrix. Rev. Bras. Entomol. 54, 339-349.

Hamed, K.H., 2008. Trend detection in hydrologic data: The Mann-Kendall trend test under the scaling hypothesis. J. Hydrol. 349, 350-363. doi:10.1016/j.jhydrol.2007.11.009

Holmes, P., 2013. Tsetse-transmitted trypanosomes Their biology, disease impact and control. J. Invertebr. Pathol. 112, S11-S14.

Holsten, A., Vetter, T., Vohland, K., Krysanova, V., 2009. Impact of climate change on soil moisture dynamics in Brandenburg with a focus on nature conservation areas. Ecol. Modell. 220, 2076-2087. doi:10.1016/j.ecolmodel.2009.04.038

Kendall, M.G., 1976. Rank Correlation Methods, 4th ed. Griffin.

Kisi, O., Ay, M., 2014. Comparison of Mann - Kendall and innovative trend method for water quality parameters of the Kizilirmak River, Turkey. J. Hydrol. 513, 362-375. doi:10.1016/j.jhydrol.2014.03.005

Kitron, U., Otieno, L.H., Hungerford, L.L., Odulaja, A., Brigham, W.U., Okello, O.O., Joselyn, M., Mohamed-Ahmed, M.M., Cook, E., 1996. Spatial analysis of the distribution of tsetse flies in the Lambwe Valley, Kenya, using Landsat TM satellite imagery and GIS. J. Anim. Ecol. 65, 371-380.

Lawler, J.J., White, D., Neilson, R.P., Blaustein, A.R., 2006. Predicting climate-induced range shifts: model differences and model reliability. Glob. Chang. Biol. 12, 1568-1584.

Matawa, F., Murwira, A., Schmidt, K.S., 2012. Explaining elephant (Loxodonta africana) and buffalo (Syncerus caffer) spatial distribution in the Zambezi Valley using maximum 
entropy modelling. Ecol. Modell. 242, 189-197. doi:10.1016/j.ecolmodel.2012.05.010

Matawa, F., Murwira, A., Zengeya, F.M., Atkinson, P.M., 2016. Modelling the spatialtemporal distribution of tsetse (Glossina pallidipes) as a function of topography and vegetation greenness in the Zambezi Valley of Zimbabwe. Appl. Geogr. 76, 198-206. doi:10.1016/j.apgeog.2016.09.008

Matawa, F., Murwira, K.S., Shereni, W., 2013. Modelling the Distribution of Suitable Glossina Spp. Habitat in the North Western parts of Zimbabwe Using Remote Sensing and Climate Data. Geoinformatics Geostatistics An Overv. S1, S1-S016. doi:10.4172/2327-4581.S1016

Munang'andu, M.H., Siamudaala, V., Munyeme, M., Nalubamba, K.S., 2012. A Review of Ecological Factors Associated with the Epidemiology of Wildlife Trypanosomiasis in the Luangwa and Zambezi Valley Ecosystems of Zambia. Interdiscip. Perspect. Infect. Dis. 2012. doi:10.1155/2012/372523

Parolo, G., Rossi, G., Ferrarini, A., 2008. Toward improved species niche modelling: Arnica montana in the Alps as a case study. J. Appl. Ecol. 45, 1410-1418. doi:10.1111/j.13652664.2008.01516.x

Pearson, R.G., 2006. Climate change and the migration capacity of species. Trends Ecol. Evol. 21, 111-113. doi:10.1016/j.tree.2005.11.022

Pearson, R.G., Thuiller, W., Araújo, M.B., Martinez-Meyer, E., Brotons, L., McClean, C., Miles, L., Segurado, P., Dawson, T.P., Lees, D.C., 2006. Model-based uncertainty in species range prediction. J. Biogeogr. 33, 1704-1711. doi:10.1111/j.13652699.2006.01460.x

Pender, J., Mills, A., Rosenburg, L., 1997. Impact of tsetse control on land use in the semi-arid zone of Zimbabwe. Phase 2: Analysis of land use change by remote sensing imagery. Natural Resources Institute, Chatham.

Peterson, A.T., Papes, M., Eaton, M., 2007. Transferability and model evaluation in ecological niche modeling: a comparison of GARP and Maxent. Ecography (Cop.). 30, 550-560. doi:10.1111/j.2007.0906-7590.05102.x

Phillips, S.J., Anderson, R.P., Schapire, R.E., 2006. Maximum entropy modeling of species 
geographic distributions. Ecol. Modell. 190, 231-259. doi:10.1016/j.ecolmodel.2005.03.026

Phillips, S.J., Dudik, M., 2008. Modeling of species distributions with Maxent : new extensions and a comprehensive evaluation. Ecography (Cop.). 31, 161-175. doi:10.1111/j.2007.0906-7590.05203.x

Rutkowska, A., 2015. Properties of the Cox-Stuart Test for Trend in Application to Hydrological Series: The Simulation Study. Commun. Stat. - Simul. Comput. 44, 565579. doi:10.1080/03610918.2013.784988

Scoones, I., Dzingirai, V., Anderson, N., MacLeod, E., Mangwanya, L., Matawa, F., Murwira, A., Nyakupinda, L., Shereni, W., Welburn, S.C., 2017. People, Patches, and Parasites: The Case of Trypanosomiasis in Zimbabwe. Hum. Ecol. 45, 643-654. doi:10.1007/s10745-017-9929-y

Sibanda, M., Murwira, A., 2012. Cotton fields drive elephant habitat fragmentation in the Mid Zambezi Valley, Zimbabwe. Int. J. Appl. Earth Obs. Geoinf. 19, 286-297.

Thuiller, W., 2004. Patterns and uncertainties of species' range shifts under climate change. Glob. Chang. Biol. 10, 2020-2027. doi:10.1111/j.1365-2486.2004.00859.x

Van Den Bossche, P., de La Rocque, S., Hendrickx, G., Bouyer, J., 2010. A changing environment and the epidemiology of tsetse-transmitted livestock trypanosomiasis. Trends Parasitol. 26, 236-243. doi:10.1016/j.pt.2010.02.010

Yan, Y., Yang, Z., Liu, Q., 2013. Nonlinear trend in streamflow and its response to climate change under complex ecohydrological patterns in the Yellow River Basin, China. Ecol. Modell. 252, 220-227. doi:10.1016/j.ecolmodel.2012.05.022

Yates, C.J., Elith, J., Latimer, A.M., Maitre, D. LE, Midgley, G.F., Schurr, F.M., West, A.G., 2010. Projecting climate change impacts on species distributions in megadiverse South African Cape and Southwest Australian Floristic Regions: Opportunities and challenges. Austral Ecol. 35, 374-391. doi:10.1111/j.1442-9993.2009.02044.x 